\title{
Trends and Issues in 5G Networking and Beyond
}

\author{
Koichi Asatani
}

Nankai University, Tianjin, China

E-mail: asatani@ieee.org

Received 19 March 2018;

Accepted 30 March 2018

\begin{abstract}
$5 \mathrm{G}$ is being discussed and developed related to almost all areas of information and communications of the future. The Internet is evolving from humanto-human communications to machine to machine communications, which resulted in Internet of Things (IoT). IoT will drastically reshape the framework of business, industries, administration, and many other areas of the society. This article reviews trends in Global ICT and clarifies that 5G is a kind of goal in many ways in research and development on ICT. It describes the expected $5 \mathrm{G}$ images and discusses the issues in realization and in deployment as an infrastructure.
\end{abstract}

Keywords: 5G, IoT, Converged Network, SDN, NFV, Network Slice, FMC, Universal Services, Network Neutrality.

\section{Introduction}

Mobile communications are playing a leading role in ICT for decade. Global mobile phone subscriptions exceeded fixed phone subscriptions in 2001, is over seven billion as of 2017, and growing further.

Mobile access has been enhanced through higher bandwidth utilization efficiency resulting in higher and higher data rate. The maximum data rate of

Journal of ICT, Vol. 5_3, 203-224. River Publishers

doi: $10.13052 /$ jicts2245-800X.531

This is an Open Access publication. (c) 2018 the Author(s). All rights reserved. 
$3 \mathrm{G}$ is $20 \mathrm{Mbps}$ up to $100 \mathrm{Mbps}$ and is enhanced to $100 \mathrm{Mbps}-1 \mathrm{Gbps}$ in $4 \mathrm{G}$. In $3 \mathrm{G}$ and $4 \mathrm{G}$, the main focus was to enhance access data rate.

Now $5 \mathrm{G}$ is being developed. The $5 \mathrm{G}$ is not only further enhancement of mobile access data rate. Its target is to cover diversified requirements as infrastructure for multimedia and IoT as well as voice communications.

This article addresses ICT trends, and historical review of network technology. It also will touch on issues on $5 \mathrm{G}$ and future networks.

\section{Paradigm Shifts in ICT}

\subsection{Global Trends and Issues in ICT}

The communication media are shifting from legacy telephony to emerging data services in many ways.

Services shifted from human communication oriented services to machineoriented services; human-to-human, then human-to-machine, and finally machine-to-machine communications. Machine-to-machine communications will involve much diversified traffic types, such as stable but broadband streaming type of traffic and very sparse intermittent narrowband type of traffic.

Also, latency was not a critical issue in 4G, although latency affects QoS in voice communication. Connected and autonomous cars will require ultra-very low latency in communications for some critical use cases.

Subscription of fixed line communications was exceeded by that of mobile communications, and finally fixed and mobile communications are converged as FMC (fixed and mobile convergence) in NGN, which was installed in 2008.

Voice communications are still indispensable and the major player was shifted from fixed telephone to mobile telephone. The number of mobile phone subscribers exceeded the number of fixed phone subscribers in 2001. Fixed telephone subscriptions have its peak in 2006 and are still gradually decreasing. Other subscriptions except fixed telephone are still growing.

These paradigm shifts will pose different requirements to network capabilities. A converged network will be a kind of solution, which can support any type of communication modes, narrowband and broadband, streaming type of communication, and massive but very intermittent communications. Traffic engineering for a converged network will require different approach; from static traffic engineering approach to adaptive traffic engineering approach. The network will also integrate IoT applications. IoT devices will be very 


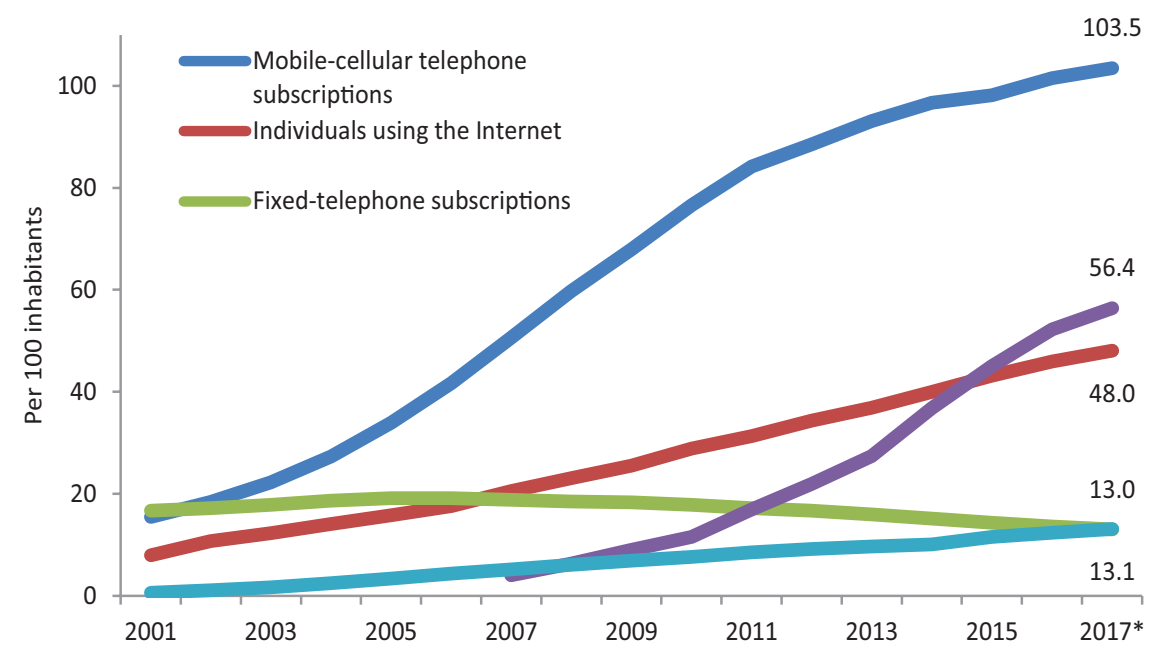

Note: * Estimate

Figure 1 Global ICT developments, 2001-2017 [1].

massive and densely/thinly distributed and will generate high speed and very low speed data.

The hot topics in ICT include Internet of Things (IoT), M2M, SDN/NFV, cloud computing, connected cars, smart city, smart factory, smart house, smart agriculture, etc.

The global trends of ICT developments are shown in Figure 1 [1].

\subsection{Mobile Network Evolution}

Access portion is one of bottle necks of mobile communication throughput in data applications and has been one of the main topics in mobile technologies. In $1 \mathrm{G}$ (analog) and $2 \mathrm{G}$ (first digital generation), voice communication was the main application. The available data rates are around $2 \mathrm{kbps}$ in $1 \mathrm{G}$ (Analog) and $14.4 \mathrm{kbps}-64 \mathrm{kbps}$ in $2 \mathrm{G}$ networks. The focus of $1 \mathrm{G}$ and $2 \mathrm{G}$ was to enable a call from anywhere to anywhere and enhance bandwidth utilization in order to accommodate more channels with the same bandwidth.

In $3 \mathrm{G}$ (voice plus data) and 4G (data plus voice on data), data and multimedia applications were evolving and the main focus was shifted to the increase in data rate. The maximum data rate in $3 \mathrm{G}$ is $20 \mathrm{Mbps}-100 \mathrm{Mbps}$, and 


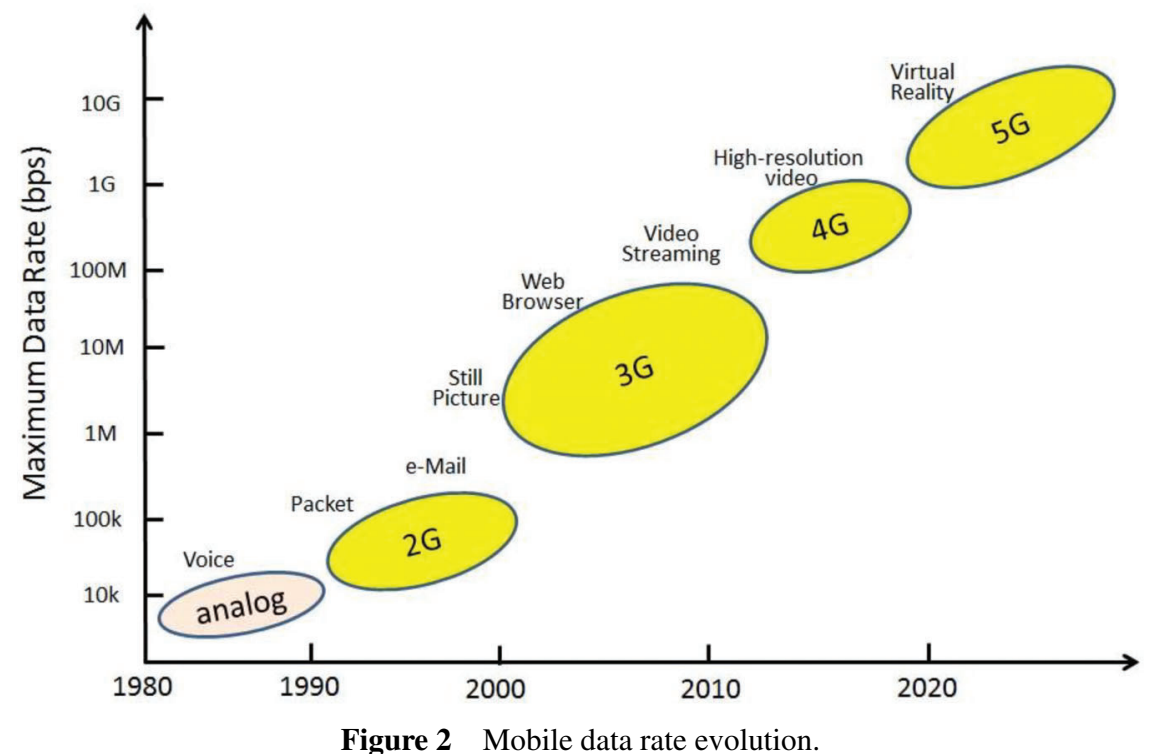

that of $4 \mathrm{G}$ is $100 \mathrm{Mbps}-1 \mathrm{Gbps}$. The data rate trends in mobile access systems are summarized in Figure 2.

\subsection{Internet of Things (IOT) - Cellular and Non-cellular loT}

The Internet applications include e-mail, web-browsing, e-commerce, IP telephony, video streaming and cloud services. These applications are human-to-human, and human-to-machine type of communications. Machineto-machine (M2M) communications will enable wider applications, such as sensor control, and remote machine control. Based on M2M, IoT is under development in many areas. Value of IoT applications will be enhanced with machine learning or deep learning by deploying artificial intelligence. IoT will reshape the future business, industrial and societal activities by enabling and enhancing operational efficiency, quality control, quick decision-making and smart relationships with customers through M2M applications.

Major IoT wireless systems are summarized in Figure 3. IoT systems are classified into two categories; non-cellular IoT and cellular IoT.

Non-cellular IoT systems, such as Sigfox, LoRa/LoRaWAN, and IEEE WiFiHaLow, use unlicensed band and will require a new non-cellular access points and associated network infrastructure [2-5]. 
Trends and Issues in 5G Networking and Beyond 207

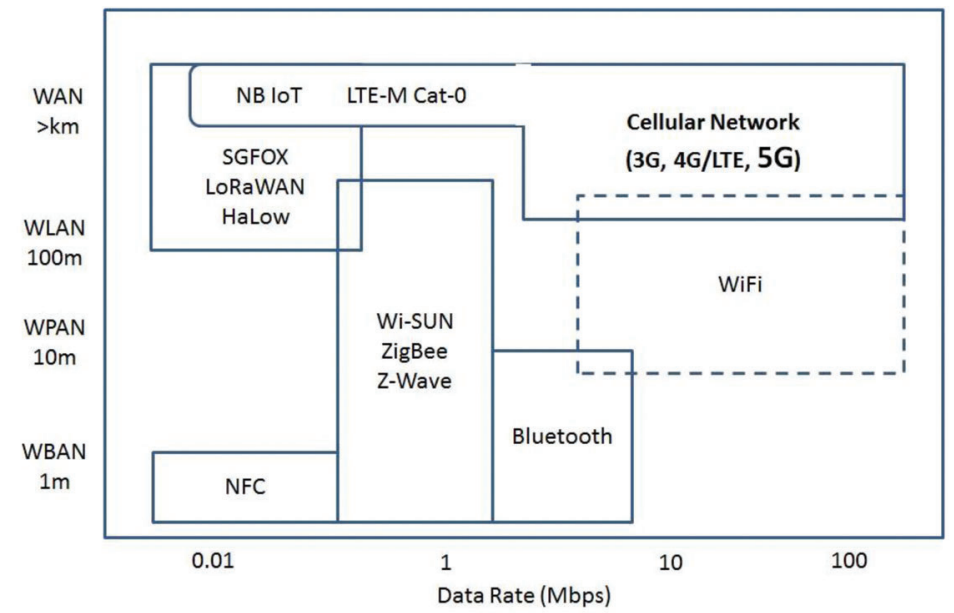

NB-IoT: Narrowband IoT, Cat-0: LTE UE category 0, LTE-M: LTE UE category M1

Figure 3 IoT wireless systems.

Cellular IoT, such as LTE UE category 0 (Cat-0), LTE UE category M1 (LTE-M) and Narrowband IoT (NB-IoT), uses licensed band and will be able to utilize a cellular network, which features very wide or nation-wide coverage. LTE-M is compatible with the existing LTE network. NB-IoT can be deployed in-band and use the resource blocks within an LTE carrier as well as those in the guard-band [6-8].

Quality of service of cellular IoT is manageable, different from noncellular IoT. In operation in licensed spectrum, which is under regulation, quality of services is guaranteed which operates in managed frequencies and bands. For the unlicensed non-cellular IoT, it will be harder to guarantee QoS, as there will be other equivalent unlicensed and unmanageable networks operating on the same frequencies, causing possible interference.

Also, in non-cellular IoT, transmit power or duty cycle for networks operating in the unlicensed bands are restricted as there are other unlicensed networks, not manageable. Higher transmit power in cellular IoT enables larger cell size or fewer cells to cover the same area and will reduce the deployment cost. Additionally, existing cellular network infrastructure can be used for cellular IoT, and it may require lower deployment costs.

With these features, cellular IoT is expected to be a big stream in the future. Currently, cellular IoT is introduced in such use cases as shared bicycles and connected cars on top of $3 \mathrm{G}$ and $4 \mathrm{G}$ networks. 


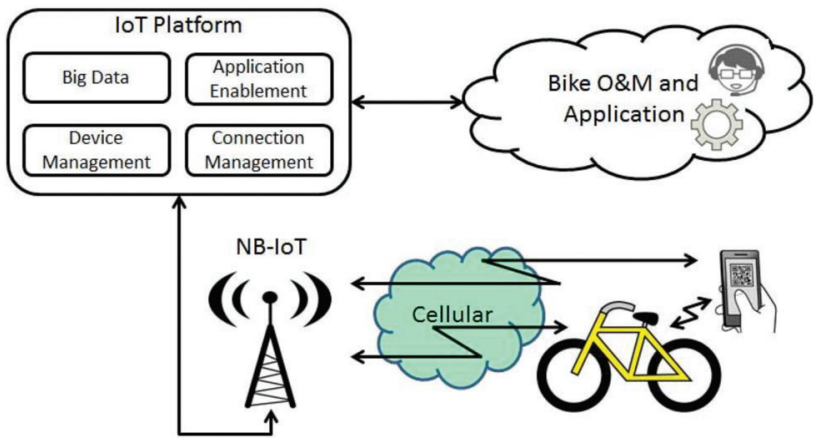

Figure 4 NB-IoT Enabled Smart Shared Bike Locks and Latency [9].

Bicycle sharing is growing very rapidly. For example, in China, shared bicycles are used by more than 32 million users every month on average, reaching coverage of $8.04 \%$ in first-tier cities including Beijing, Tianjin, and Shanghai. Currently, 3G-based smart locks are widely used in China [9].

NB-IoT-based shared bike locks provide lower power consumption, better coverage, and lower latency and are being introduced. Users unlock a shared bike with lower latency than GSM based shared bike locks. An NB-IoT smart lock can be unlocked within 2 seconds, whereas 6.81 seconds by GSM lock. Smartphone accesses to IoT device of the shared bike through Bluetooth and accesses to Application to unlock through NB-IoT. After authentication of the smartphone, the shared bike is unlocked remotely. The system configuration is shown in Figure 4 [9].

Connected car penetration in USA, for example, is estimated to be 19.3 and $72.4 \%$ in 2017 and in 2022 [10].

Presently, the dominant technology in the wide-area segment is $3 \mathrm{G}$. However, by 2023 , IoT cellular connectivity will mainly be provided by $4 \mathrm{G}$ (LTE) and 5G. The majority of these connections will be over LTE networks, while 5G technology will continue to support an increase in IoT applications, especially those requiring critical communications. $5 \mathrm{G}$ will also provide rapid and cost-effective provisioning of new IoT services.

Based on technologies like Cat-M and NB-IoT, a growing number of cellular IoT networks are being deployed, with more than 20 networks commercially launched across several regions [11].

The firmware upgradability is also important aspect of IoT from viewpoints of security. 


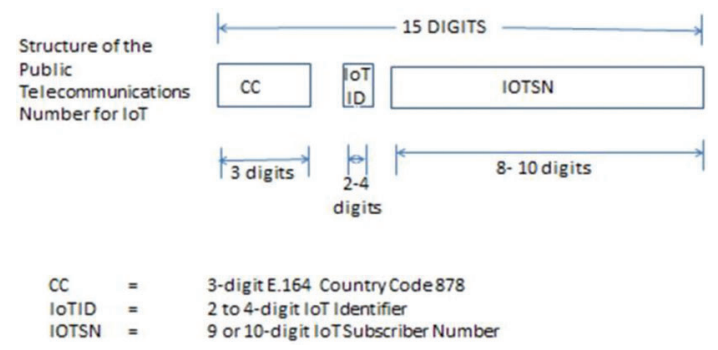

Figure 5 IoT Number Structure [14].

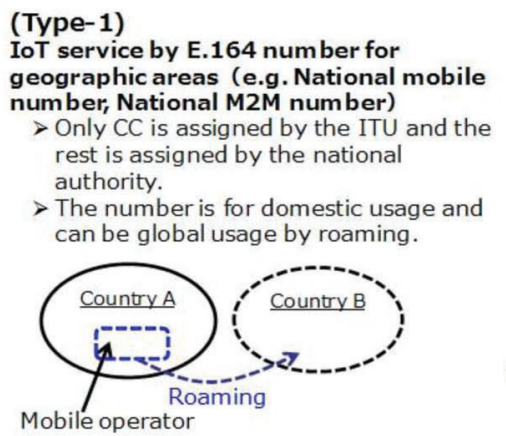

(Type-2)

IoT service by E.164 number for global services/networks (e.g.global IoT/M2M number )

$>$ The number is assigned by ITU.

$>$ The number is for global usage.

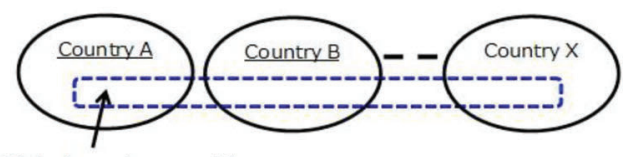

Global service provider for IoT/M2M

Figure 6 Global IoT/M2M services by two kinds of numbering scheme [14].

\subsection{Identification in IoT}

SIM embedded in cellular IoT devices will be assigned a telephone number or/and IP address as device ID.

Telephone numbering plan principle is defied in ITU-T Rec. E.164 [12]. Its application to mobile numbering is defined in ITU-T Rec. E.212 [13]. The international numbering plan specifies maximum fifteen digit format in Rec. E.164. The mobile numbering plan is defined in Rec. E. 212, under the umbrella of Rec. E.164. If telephone numbering plan is deployed as device ID, the overall numbering plan has to follow ITU-T Rec. E.164 and Rec. E.212. IoT number structure is shown in Figure 5 [14].

Two types of IoT by Rec. E.164 are defined as in Figure 6 [14]. Type1 IoT service by Rec. E.164 number is defined as national mobile number and national M2M number. Only country code (CC) is assigned by ITU and the rest part is assigned by the national authority. But the number is limited to domestic usage and can be globally used by roaming. Type-2 IoT service 
by Rec. E.164 number is defined for global services/networks, e.g., global IoT/M2M number. The number is assigned by ITU-T and is for global usage.

The actual number assignment for domestic part is under national regulation. In Japan, the prefix (Mobile Network Code) 020 is assigned as IoT numbering, for example [15].

IPv6 address has more capacity than telephone numbers and will be used more widely in the future. But IPv6 deployment is currently not well penetrated.

\section{Architecture Evolutions to Converged Networks}

\subsection{Toward Converged Networks, -ISDN, NGN and 5G-}

The ISDN installed in early 1980's was the first challenge toward a converged network. It provides various types of end-to-end digital connectivities through a unified User-Network Interface (UNI). It is a direct enhancement of access capability to support both of circuit mode and packet mode services based on 64kbps switched telephone network. Then, enhancement to broadband capability was challenged as B-ISDN (Broadband aspects of ISDN) by adopting a new Asynchronous Transfer Mode (ATM). The ATM is based on label switching technology with a very short ATM cell size and provides a unified transfer mode by integrating circuit mode and packet mode services through fully integrated ATM-UNI [16].

ATM was the first challenge to support any type of services, circuit mode and packet mode, narrowband and broadband services, by a single ATM technology including unified broadband UNI. The ATM was installed in core networks, but was not deployed as user-services. The network convergence trends are shown in Figure 7.

The commercial Internet access was first supported by dial up through telephone network with use of modems up to $56 \mathrm{kbps}$, in early 1980's [17]. In late 1980's, always-on access was then deployed through ISDN UNI, which supports two 64kbps channels, called B-channels, plus 16kbps D-channel simultaneously.

Broadband accesses such as ADSL, Cable and FTTH were installed since 1990's. Broadband streaming and real-time applications such as video, IP TV and IP telephony were enabled in the broadband Internet.

Broadband Internet traffics grew very rapidly and exceeded voice traffic. From network operators' view, Operation Expenditure (OPEX) is very important. Converged network is a candidate solution. Instead of building 


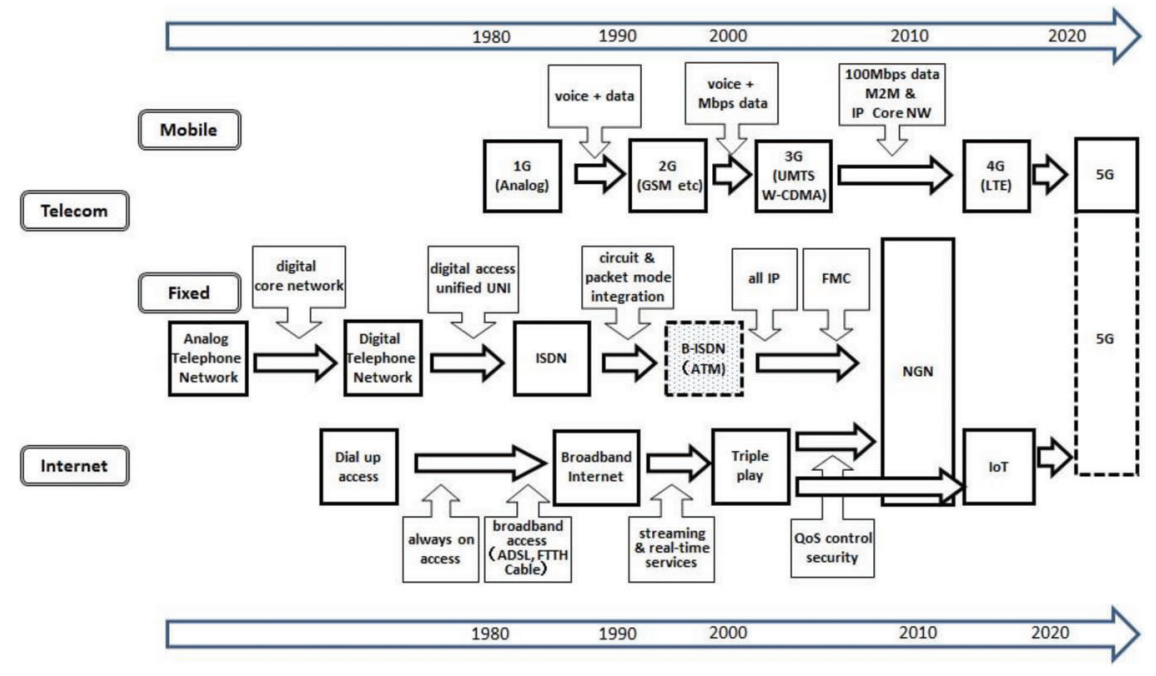

Figure 7 Network Evolutions \& Convergence.

multiple service dedicated networks, each of which requires each OPEX, a converged network will reduce OPEX in total.

The converged network deploying Internet Protocol was developed to support these data as well as voice applications. It is called "all IP network". Based on all IP network concept, NGN (Next Generation Network) was developed, which is globally standardized in ITU-T to ensure global connectivity [18].

\subsection{NGN -AII IP network Architecture}

The number of Internet users was growing very rapidly since 1990. Thanks to the increase in broadband access rate as well as the expansion of Internet users, IP data and multimedia traffics are drastically growing. More diversified applications have been developed to respond more and more diversified customers' demand. Thanks to the wireless access development, demand for mobility has been also increasing.

To respond these new trends, network operators' demand for large-capacity converged network with low OPEX was increasing with maintaining future compatibility.

Telecom carriers chose IP networks as a unified transport platform to support data and multimedia applications as well as voice. IP was adopted because IP data is dominant traffic. 


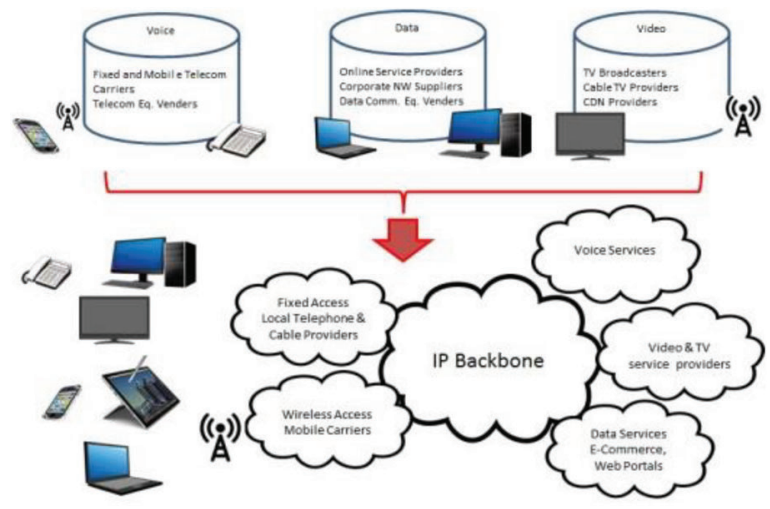

Figure 8 Architecture for All IP Integration.

For example, total IP data traffic through mobile access is approximately 44 times larger than total voice traffic in 2017 in Japan [19]. Therefore, NGN was designed as a converged network solution deploying Internet Protocol, and was globally standardized in order to ensure global connectivity. Conceptual architecture is illustrated in Figure 8. Fixed and mobile terminals access to IP backbone through fixed access network and mobile access network and enjoy triple play services, i.e., telephone, TV broadcast and Internet.

NGN also enables fixed-mobile-convergence (FMC), taking into account customers demand for more flexible mobility. It is a convergent approach from fixed network to integrate mobile access. Core network is a fixed network in fixed telephone network and also in mobile network.

\subsection{Network Virtualization/Software Defined Network}

In 2009, ITU-T initiated the study on Future Networks as the next step after NGN (Next Generation Network). Focus Group on Future Networks (FGFN) was established to identify key elements of Future Networks related standardization for two years. The identified key elements include [20]:

- Network Virtualization (NV)

- Energy Saving of Networks

- Identifications in future networks

- Data aware networking

- Software Defined Networks (SDN)

The detail study on future networks was conducted in ITU-T Study Group 13 and was resulted in ITU-T Y.3000-series Recommendations [21]. 
Network Virtualization (NV) is defined by the ability to create multiple logical, virtual networks that are decoupled from the underlying shared physical network resources. It can realize multi-virtual networks each of which responds to multi-tenancy requirements independently.

There are two types of network virtualization; internal and external network virtualizations. Internal network virtualization is the process of creating one or more logical networks by defining logical network elements such as switches and routers. External virtualization allows aggregation of multiple resources and makes the aggregated resources appear as a single resource.

Virtualization itself is not a new idea. Virtualization in computers is very common today. The computer configuration started with a vertical integrated computer including hardware, operating systems and application software, all of which are proprietary to the specific machine in the very early stage. Then, interfaces between hardware and operating system, and operating system and applications were defined. An operating system can be installed on different hardware, and applications run on top of the operating system. This makes computer easy to evolve, allowing upgrade to better operating system with maintaining the hardware, and to better applications with maintaining the operating system and vice versa.

The powerful CPU enables multiple guest operating systems (guest OSs) run on the host OS or hypervisor in a computer. The single machine works as multiple virtual computers running on a single hardware. In other words, a virtual machine is an emulation of a real or physical computer which runs on top of an operating system and can host applications, as shown in Figure 9 [22].

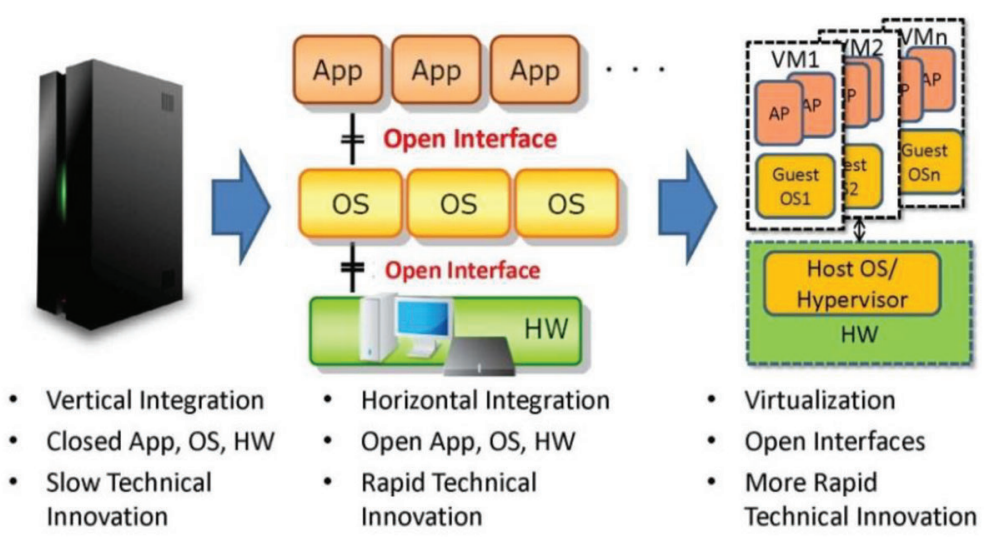

Figure 9 Computer/Server Virtualization (based on [23]). 


\section{K. Asatani}

External virtual networks were already deployed in business areas, such as CES (Centralized Extension System), which is virtual PBX by using a public exchange system in telephone office, VPN on IPNetwork, VLAN, and MVNO.

An internal virtual network connects two or more virtual machines on a single server and allows data exchanges between virtual machines in the network through virtual network interface cards on the virtual server to get higher network resource usage by minimizing traffic on the physical network. It gives a network administrator flexibility to combine virtual network elements in any way to create a network of any size and scope for the organization or create multiple networks that will share the same physical network resources. These multiple networks are called network slices.

Internal virtualization achieves low Capital Expenditure (CAPEX) and OPEX due to its easy network operation and administration. But it creates problems such as high risk infect on logical networks which run on top of the same physical entity due to physical faults, scalability, and workload balancing and migration within network. Therefore, dependability requirements to physical entities should be very high for supporting various types of QoSs and high dependability, which are very common requirements to public infrastructure. Also, traffic engineering will be more complicated.

SDN is a kind of straight forward extension of virtual machines. Network elements such as switches and routers consist of two functions, control and forwarding functions. These functions are configured proprietary in the conventional networks. The separation of these two functions by a standardized interface, if possible, makes the network more evolvable and controllable.

SDN is defined as the network where control plane of the network and forwarding plane are separated with the standardized interface in-between two planes. Therefore it enables more efficient orchestration and automation of network services through centralized policy-enabled work-flow automation. Openflow network configuration is shown in Figure 10, as an example of SDN [23]. The interface indicated as OpenFlow protocol in Figure 10 is newly defined between control plane and data plane (user plane).

The conceptual architecture of network virtualization is shown in Figure 11 [24]. Network virtualization realizes independent virtual networks, which are called logically isolated network partitions (LINPs) as shown in Figure 12. Multiple virtual (network) resources share a single physical (network) resource. An n-th set of virtual (network) resources forms an n-th virtual network (LINPn). The virtual network resources are managed by the virtual resource manager (VRM). The virtual network LINPn is managed by 


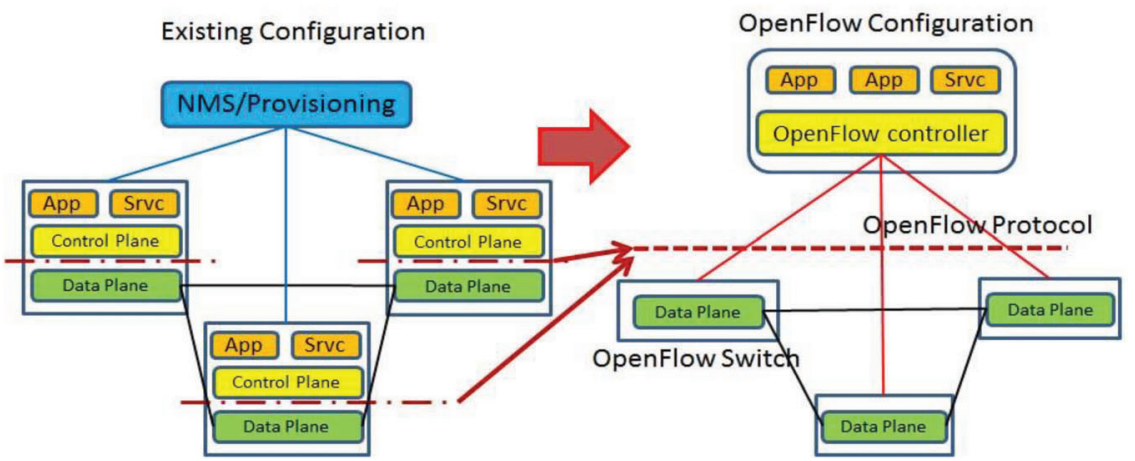

Figure 10 OpenFlow Network.

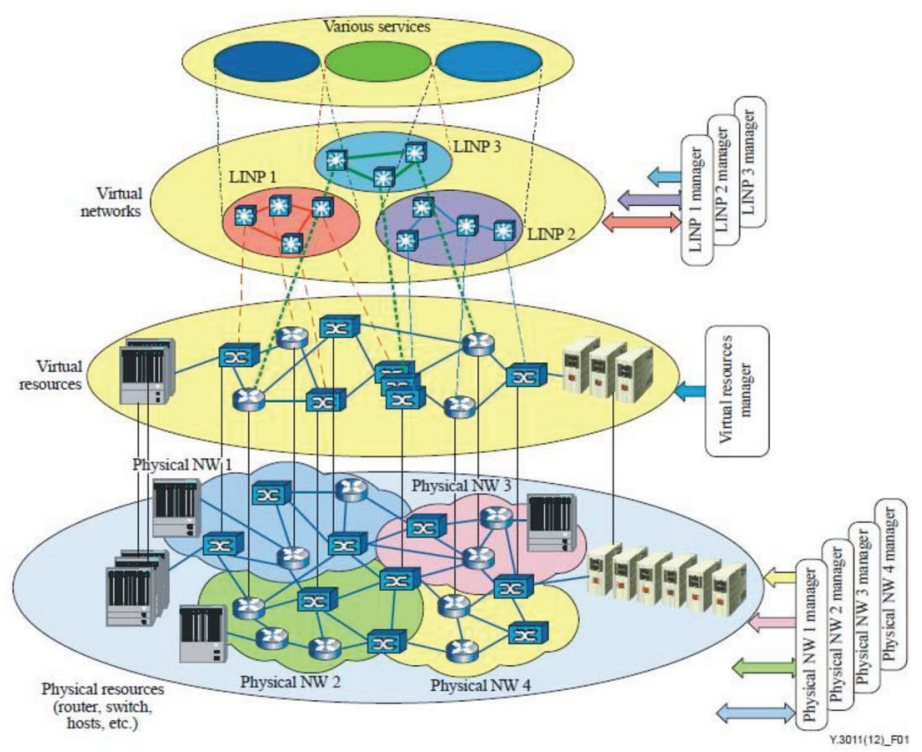

Figure 11 Conceptual architecture of network virtualization.

LINPn manager [24]. On top of each LINP, dedicated service supported by LINP is provided.

Network slicing is a way to provide an LINP per users' requirements. LINPs are constructed based on the users' requirements. If an appropriate LINP type is not provisioned, a new LINP could be created per users' demand. The requirements, based on the administration policy, are delivered to the VRM which coordinates the allocation of LINPs so that appropriate LINP 


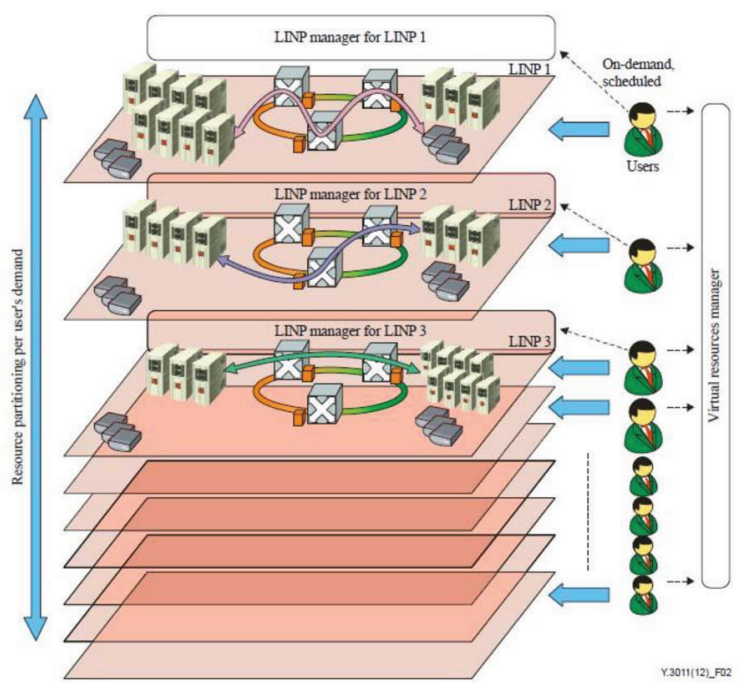

Figure 12 Network Slicing [24].

is provided to users. The VRM, by controlling all virtual resources, creates an LINP manager, as shown in Figure 12. An LINP has various characteristics, such as partitioning, isolation, abstraction, flexibility or elasticity, programmability, authentication, authorization, and accounting [24].

Architecture of LINP and LINP manager is based on the SDN concept, separating data plane (LINP) and control plane (LINP manager).

\section{Perspective and Issues of 5G}

\subsection{G Use cases}

Expected use cases of $5 \mathrm{G}$ are much diversified. The requirements to $5 \mathrm{G}$ are coming from diversified $5 \mathrm{G}$ use cases.

$5 \mathrm{G}$ will be a major platform with the enhanced speed after $4 \mathrm{G}$. $5 \mathrm{G}$ use cases have been investigated. They are, for example [25],

- Broadband in dense areas

- Broadband access everywhere

- Higher user mobility, such as bullet trains

- Massive Internet of Things, such as sensor networks

- Extreme real-time communications, such as autonomous cars

- Lifeline communications, such as in disasters 


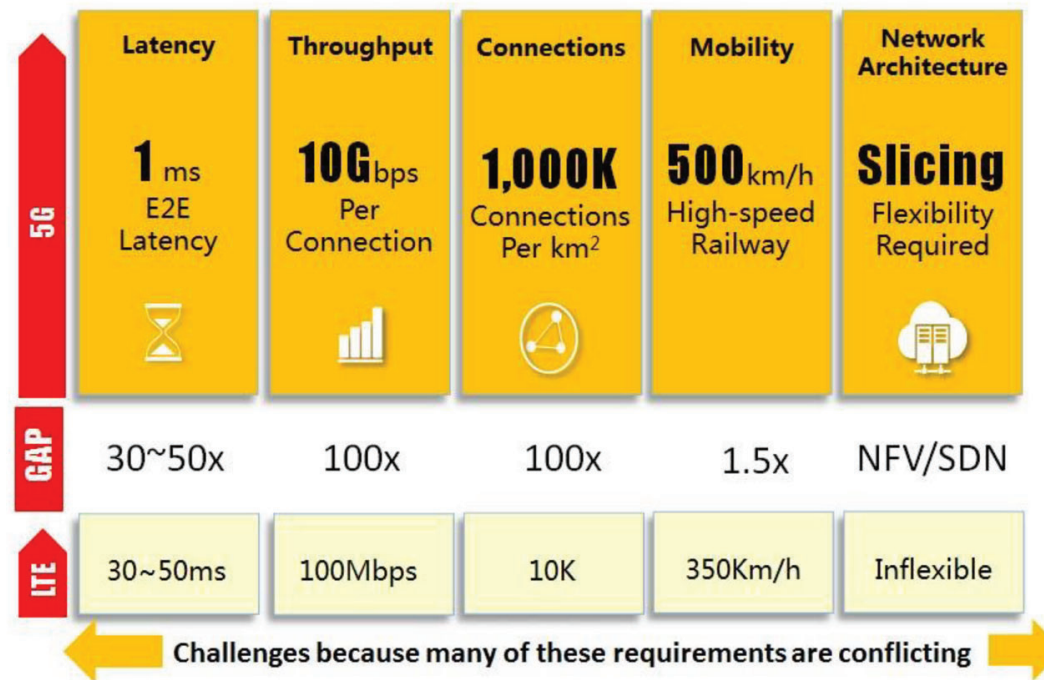

Figure 13 Diversified Challenges and Gaps to 5G [26].

- Ultra Reliable communications, such as in e-Health

- Broadcast-like services, such as broadcast emulations

\subsection{G Target and Challenges}

To achieve these use cases, target bandwidth is greater than $1 \mathrm{Gbps}$, e.g., $10 \mathrm{Gbps}$. The target number of connections $/ \mathrm{km}^{2}$ of $5 \mathrm{G}$ is 100 times larger than 4G. Mobility of $500 \mathrm{~km} / \mathrm{s}$, ultra- low latency of less than $1 \mathrm{~ms}$ for autonomous cars, flexibility for support low speed data but high density and massiveness of IoT. Target of 5G and the current state of art are compared in Figure 13 [26].

These requirements are very hard to be achieved by a single network, or single policy. Then three typical network slices are planned to work on a single physical network. One for high-speed multimedia, one for low latency applications, and one for IoT, dense connections as shown in Figure 14 [27].

An example of $5 \mathrm{G}$ network slices realization is shown in Figure 15, which illustrate how virtualized networks functions are allocated in each network slice in an edge cloud/node and in a central cloud/node.

Typically, at least three network slices are supposed to be implemented for mission critical IoT applications, massive IoT with very low speed data and ultra-broadband video streaming. The mission critical IoT applications include autonomous cars associated with connected cars. The number of network 


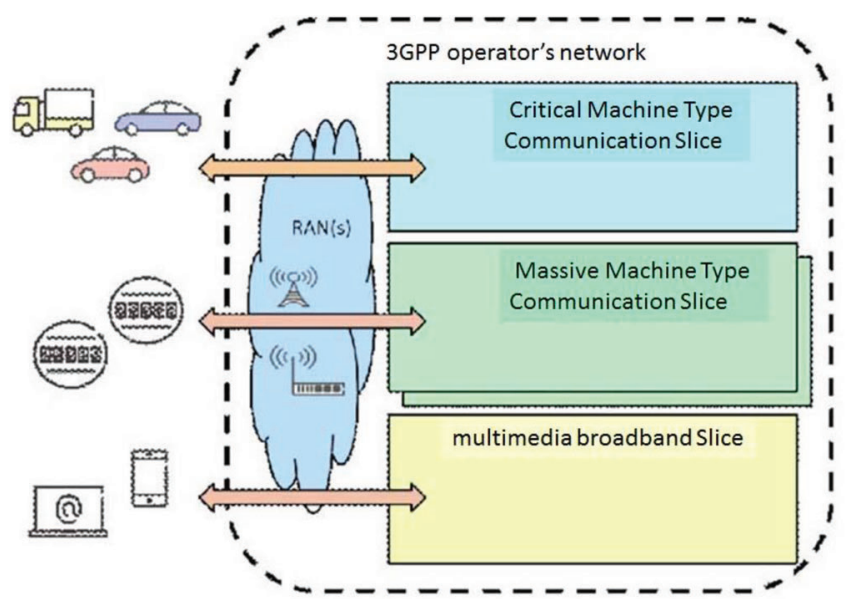

Figure 14 5G Architecture and Network Slices [27].
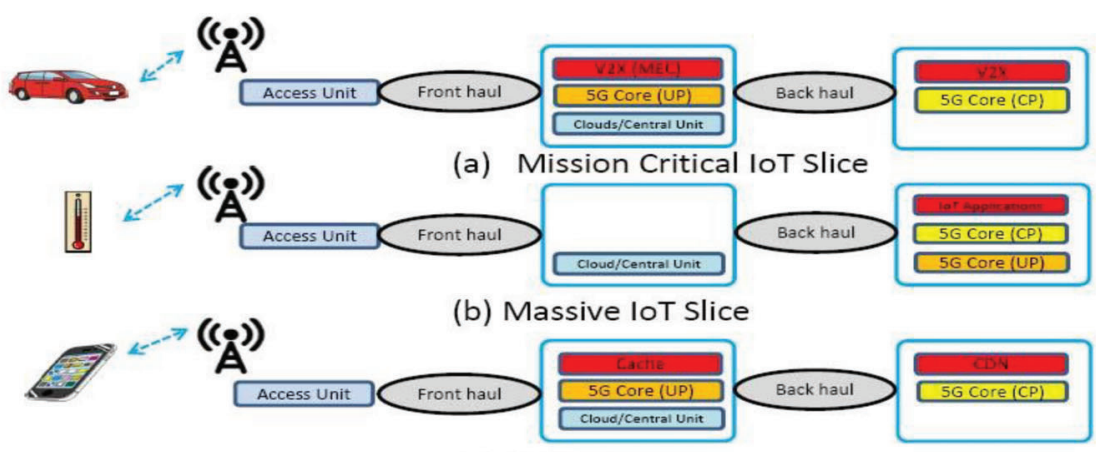

(c) UHD Slice

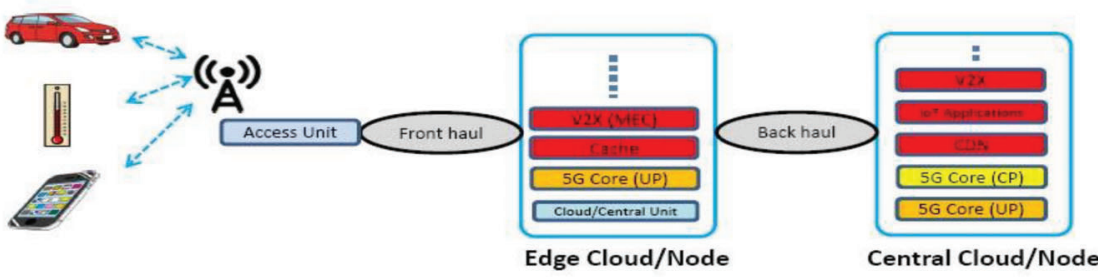

(d) Physical Realization

MEC: Mobile Edge Computing, UP: User Plane, CP: Control Plane, UHD: Ultra High Definition TV

Figure 15 An Example of Virtualized Function Allocations in Each Slice. 
slices is not limited to three. If emerging environments and applications require different policy from those of the three slices' policies, the flexible 5G architecture will allow implementing new network slices with different policies.

\section{Regulatory and Other Issues}

\subsection{Regulatory Aspects on Dependability as Infrastructure}

Band licensing is a main issue in $5 \mathrm{G}$ access, but is not dealt with in this article. This chapter mainly discusses regulatory issues other than the band licensing. Regarding the battle in band licensing can be found in [28], for example.

Telecommunications common carriers are regulated as one of the key social infrastructures. In particular, public telephony services are supposed to follow the severe requirements. It should be public, i.e., the service is open to anyone. It should support universal service, and minimize divides. It should be very dependable. The dependability of the service should be more than five nines (99.999\%). Other aspects are also required such as business sustainability, QoS guarantee, even under competitive environments as seen in many countries. In addition to these requirements, it should be responsible for R \& D for future technology and emerging environments.

Service availability of five nines requires very dependable physical entity for being used as a physical infrastructure layer in network slicing. Protection schemes managed by forwarding plane and control plane are requested in achieving the target dependability level.

To satisfy "Five Nines" high availability standards, and provisioning is important for very fast fault recovery through redundancy. Target downtime is less than $5 m 15 s$ in a year.

As a social infrastructure, large scale faults in size and long duration length are strictly regulated. Once such an instance happened to infect large number of customers for long time duration, carriers should report to regulating national authority. In Japan, for example, more than two hours outage duration affecting more than 30,000 customers should be reported to the government [29]. In USA, such reporting threshold is that the outage duration over 30 minutes and the number of "user-minutes" potentially affected per outage must equal or exceed 900,000 [30]. In United Kingdom, the following reporting thresholds are defined. If minimum numbers of end customers' affected and minimum duration of service loss or major disruption are 1,000 and 24 hours, 100,000 and 1 hour, and 100,000,000 and 10 minutes, the outage incidence should 


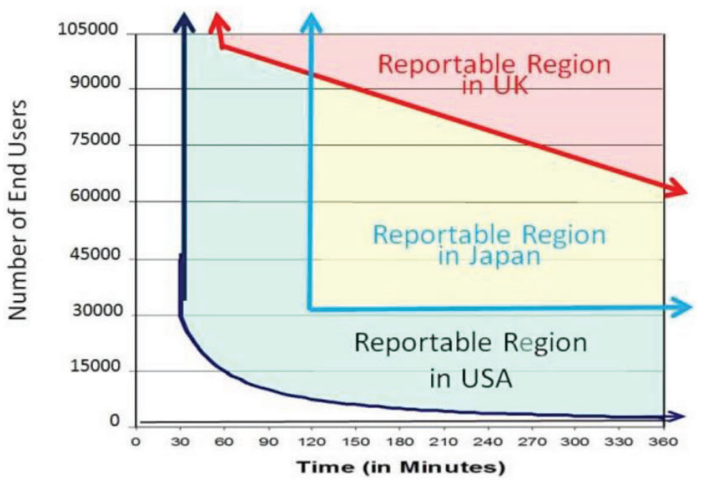

Figure 16 Threshold Criteria for Telecommunication Outage Reporting.

be reported [31]. Threshold criteria for telecommunication outage reporting in these three countries are illustrated in Figure 16. The vertical axis shows number of affected users and the horizontal axis shows outage duration in hour. The coloured areas show the reportable regions of these countries where carriers should report to the national authorities.

\subsection{Network Neutrality}

Network neutrality is a principle that service providers and governments should treat all data on the public network equally, with no discrimination or different charging by user, content, site, platform, application, type of attached equipment, and modes of communications [32].

Network neutrality is one of the on-going controversial issues unresolved. The converged networks will deal with any types of applications with very different traffic characterizations. Therefore, it will be more difficult to realize network neutrality. Furthermore, more applications called OTT are using network as dumb pipe. Then a new principle will be required to create a new reasonable balance between network, not as a dumb pipe but as a smart pipe, and applications [33].

\subsection{Universal Services}

Initially an obligation imposed on monopoly operator of voice telephony, to expand coverage to provide services in remote and underserved areas. Before competition starting in 1980's, many network operators typically crosssubsidized cost of universal service obligations with revenues from other 
services. With introduction of competition, regulators substituted this implicit cross-subsidization with requirement that all/some operators contribute a percentage of their revenues to "universal service fund".

$\mathrm{NV}$ will reform the map of players who are responsible for services, service platforms and networks. New rule should be established taking into account new market pictures under different market and regulatory environments depending on regions and countries. With use of virtual networks, it should be clarified that the primarily responsible player(s) for universal services. New rule should be established based on the clarification [34].

\subsection{Other Issues}

It will be essential to gather big data based on users' behaviours in order to achieve smart operation of many virtual networks based on the data. As a result, it will be very easy to look into the detailed information attached to individuals. Harmonious framework is essential to reasonably balance between the requirements and privacy protection, including data portability across the borders.

Smart operation also will require smart monitoring and smart reconfigurations with use of machine learning and AI [35].

Emergency call and disaster recovery mechanisms should be also incorporated in conjunction with social infrastructure.

\section{Conclusions}

This article provides brief historical review for network evolution toward 5G, which attracts big interests in networking, network software and applications in the future. To make $5 \mathrm{G}$ full reality, there are still many technical challenges to overcome, and also many regulatory issues.

Further studies are required in relations to traffic engineering for $5 \mathrm{G}$ converged networks. Great expectations are being received to the artificial intelligence, in order to resolve the issue. Network slices are independent with each other. The traffic should be monitored and managed in each network slice. The orchestration of network slices is required for harmonization between each slice characteristics and the over-all restrictions posed by physical resources.

Some operators launched plans to install the 5G in 2020 or sooner. $4 \mathrm{G}$ instalment started in 2010's, and is now penetrating globally. 4G will be used as the major platform in a decade or so. Then, $5 \mathrm{G}$ will coexist with $4 \mathrm{G}$ for a 


\section{K. Asatani}

decade or so, too. The $5 \mathrm{G}$ interworking or interconnection with $4 \mathrm{G}$ and others is a very big issue.

Global standards and harmonization are essential in accommodating diversified requirements of different countries and regions, although global connectivity is essential.

\section{References}

[1] ITU Measuring the Information Society Report 2017.

[2] LoRa Alliance, Available at: https://www.lora-alliance.org/

[3] Wi-SUN Alliance, Available at: https://www.wi-sun.org/index.php/en/

[4] Wi-Fi Alliance, Available at: http://www.wi-fi.org/ja/discover-wi-fi/ wi-fi-halow

[5] SIGFOX, Available at: http://www.soumu.go.jp/main_content/0004520 35.pdf]

[6] Cat-0, Evolved Universal Terrestrial Radio Access (E-UTRA); User Equipment (UE) radio access capabilities, 3GPP TS36.306 (Rel. 12), Sept. 2017.

[7] LTE-M, ibid (Rel. 13), Dec. 2017.

[8] NB IoT ibid (Rel. 13), Dec. 2017.

[9] Based on Huawei, Available at: http://www.huawei.com/minisite/iot/en/ smart-bike-sharing.htm]

[10] Available at: https://www.statista.com/outlook/320/109/connected-car/ united-states\#]

[11] 2017 Ericsson Mobility Report.

[12] ITU-T Rec. E.164, The international public telecommunication numbering plan, May 1997.

[13] ITU-T Rec. E.212, The international identification plan for public networks and subscriptions, Sept. 2016.

[14] Draft ITU-T Recommendation E.IoT-NNAI, Internet of Things Naming Numbering Addressing and Identifiers, Dec. 2017.

[15] Ordinance of the Ministry of Posts and Telecommunications No. 8, 1997 (in Japanese).

[16] ATM ITU-T Rec. I. 121, Broadband aspects of ISDN, 1991, and other I-series Recs.

[17] ITU-T Rec. V.90, A digital modem and analogue modem pair for use on the Public Switched Telephone Network (PSTN) at data signalling rates of up to $56000 \mathrm{bit} / \mathrm{s}$ downstream and up to $33600 \mathrm{bit} / \mathrm{s}$ upstream, Feb. 1998. 
[18] ITU-T Rec. Y. 2200 series on Next Generation Networks.

[19] By calculation based on White Paper, Information and Communications in Japan 2017 ed., July 2017.

[20] ITU-T Focus Group on Future Networks, FG-FN OD-72 Rev. 1, Draft Deliverable on "Future Networks: Design Goals and Promising Technologies", Dec. 2010.

[21] ITU-T Rec. Y.3000-Y.3499: Future networks.

[22] Y. Sato, Technical Innovation and Standardization, IEICE Society Conference 2015.

[23] Open Networking Foundation, OpenFlow Switch Errata, June 2012. Available at: https://3vf60mmveq1g8vzn48q2o71a-wpengine.netdnassl.com/wp-content/uploads/2013/07/openflow-spec-v1.0.1.pdf

[24] ITU-T Rec. Y.3011 Framework of network virtualization for future networks, Jan. 2012.

[25] NGMN, 5G White Paper, Available at: https://www.ngmn.org/5g-whitepaper/5g-white-paper.html, March 2015.

[26] Based on Peter Ashwood-Smith, 5G Wireline Challenge. SG13 Regional Workshop for Africa, Mar. 2016.

[27] 3GPP TR 22.891, 2016.

[28] National Instruments, White Paper, "mmWave: The Battle of the Bands" Available at: http://www.ni.com/white-paper/53096/en/\#toc2

[29] Ordinance for Enforcement of the Telecommunications Business Act Article 58, Paragraph 1 (in Japanese).

[30] Disruption to Communications, Federal Register vol. 69, FR 70338, Dec. 3, 2004, amended July 12, 2016.

[31] Ofcom guidance on security requirements in the revised Communications Act 2003, Feb. 2012.

[32] As an example, see https://en.wikipedia.org/wiki/Net_neutrality, although no unanimous definition.

[33] For example, Net Neutrality: What You Need to Know Now, Free Press, Available at: https://www.savetheinternet.com/net-neutrality-what-youneed-know-now

[34] ITU-T Rec. Y.3035, Service universalization in future networks, June 2015.

[35] For example, EU General Data Protection Regulation (GDPR), 2016. Enforce 2018. Available at: https://www.eugdpr.org/ 


\section{Biography}

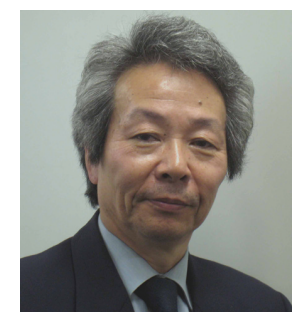

Koichi Asatani received his B.E.E.E., M.E.E.E. and Ph. D. degrees from Kyoto University in 1969, 1971 and 1974, respectively. From 1974 to 1997, Dr. Asatani was with NTT and engaged in R\&D on optical fiber communication systems, hi-definition video transmission systems, FTTH, ISDN, B-ISDN, ATM networks, IP Networks and their strategic planning. From 1997 to 2014, he served as Professor at Kogakuin University. From 1999 to 2012, he was with Graduate School of Global Information and Telecommunication, Waseda University as Visiting Professor. He is currently Chair Professor, Nankai University, Tianjin, China. He is also Professor Emeritus, Kogakuin University. He is Fellow of IEEE and Fellow of IEICE. He was appointed as Distinguished Lecturer of IEEE Communications Society for 2006-2009, 2011-2012, and 2013-2014. He served as IEEE Communications Society Director, Membership Programs Development for 2014-2015 term. He is currently Chair, IEEE Com Soc GIMS committee. He has been serving as a Board member, IEICE, and Chair, IEICE Standards Activities since 2014.

He has published more than fifty papers, authored/co-authored nineteen books, and gave more than 120 talks including keynotes and invited talks at the prestigious international conferences such as IEEE ICC and Globecom. His current interests include Information Networks including Broadband networking, Internet Interworking, IP telephony, NGN, Future Networks, IoT, 5G Networking and their QoS aspects. 